\title{
Tunisie : La mémoire des Juifs de Djerba. Yaacov Bchiri
}

\section{Luc Weissenberg}

\section{(2) OpenEdition}

Journals

Édition électronique

URL : http://journals.openedition.org/ethnomusicologie/216

ISSN : 2235-7688

Éditeur

ADEM - Ateliers d'ethnomusicologie

Édition imprimée

Date de publication : 1 décembre 2001

Pagination : 339-342

ISBN : 2-8257-07-61-9

ISSN : 1662-372X

Référence électronique

Luc Weissenberg, «Tunisie : La mémoire des Juifs de Djerba. Yaacov Bchiri », Cahiers d'ethnomusicologie [En ligne], 14 | 2001, mis en ligne le 10 janvier 2012, consulté le 30 avril 2019. URL : http:// journals.openedition.org/ethnomusicologie/216

Ce document a été généré automatiquement le 30 avril 2019.

Tous droits réservés 


\title{
Tunisie : La mémoire des Juifs de Djerba. Yaacov Bchiri
}

\author{
Luc Weissenberg
}

\section{RÉFÉRENCE}

Tunisie : La mémoire des Juifs de Djerba. Yaacov Bchiri. Enregistrements réalisés les 15 et 16 juin 2000 par André Klopmann et Vincent Zanetti à Houmt Souk, Djerba ; textes : André Klopmann ; photos : Anne-France Brunet, André Klopmann ; traduction anglaise : Isabelle Schulte-Tenckhoff. Notice bilingue français/anglais de 31 pages, 3 photos noir \& blanc. 1 CD AIMP LXIV Archives internationales de musique populaire /Musée d'ethnographie Genève, 2001.

1 Il faut souligner d'emblée l'importance de la publication d'un nouveau CD de musique judéo-arabe au Maghreb, tant ces musiques - de faible potentiel commercial - restent encore mal diffusées et donc difficilement accessibles au grand public, exception faite des grands noms du maalouf constantinois (Algérie), tels Cheikh Raymond, Reinette l'Oranaise, Lili Boniche, Alice Fitoussi, Blond-Blond ou Tahar Fergani.

2 La sortie du présent $\mathrm{CD}$ vient donc jeter une lumière particulière sur la tradition musicale des juifs de Djerba, petite île située au sud de la Tunisie, qui est encore mal connue (bien que les premiers enregistrements, réalisés par Robert Lachmann, datent de 1929). Le titre $\mathrm{du} \mathrm{CD}$ annonce ainsi bien la couleur du projet: un travail de «mémoire", à l'heure ou l'ancienne communauté juive de Djerba ne compte plus qu'un millier de membres, et que Yaacov Bchiri, le chantre-poète de la communauté depuis presque septante ans ne semble guère avoir trouvé de successeur à qui transmettre son répertoire, ses techniques de jeu, et cette mémoire du groupe qu'il a accumulée. Dans ce cadre, un tel enregistrement, in extremis, devient lui-même un maillon dans une chaîne de transmission orale, porteur d'un héritage, et certainement vecteur d'une mémoire.

3 Ainsi, le choix relativement éclectique des morceaux publiés dans ce CD reflète l'intensité de la vie religieuse et sociale de cette communauté insulaire, où la musique (et en 
particulier le chant) a un grand rôle, tant au niveau des pratiques rituelles du Shabbat, des offices quotidiens ou du pèlerinage annuel de la Ghriba, foyer de mysticisme pour juifs et musulmans, qu'au niveau des cérémonies liées au cycle de vie (naissances, circoncisions, maturité religieuse, bar-mitzvah, mariages...) et aux occasions festives, formelles ou non. Si Yaacov Bchiri est une figure centrale de la liturgie locale, il ne renie pas la tradition populaire festive, pour autant que ces genres soient clairement distincts, ce qui ne semble pas toujours être le cas. Par ailleurs, "insulaire » ne signifiant en rien " isolat », le jeu largement personnel et improvisé du chantre témoigne également des interactions de ce répertoire avec son environnement musical passé et présent, arabe (islam ibâdite), berbère, turc, puis français, mais aussi ashkénaze et israélien, donnant ainsi spontanément lieu à des « emprunts » multiformes. La «tradition » est donc poreuse, et comme ailleurs, "toute tradition musicale juive résulte d'une histoire et d'un environnement géographique particulier » (Roten 1998 : 117).

4 Aussi, la plus grande partie des pièces présentées témoigne de la double fonction de ce musicien : à la fois chantre hazzan - il est le dépositaire par excellence des répertoires liturgiques en hébreu -, il est surtout un poète-musicien-compositeur-interprète (paytan) entonnant essentiellement des poésies religieuses généralement strophiques (piyyutim) qu'il interprète assez librement (sur 18 plages, 12 sont ses compositions ou des adaptations partielles), lors de circonstances paraliturgiques. La plupart des pièces débutent ici par un prélude instrumental au 'ûd, parfois tiré du répertoire arabo-andalou traditionnel (plage 10 et 12) et, bien que l'accordage ne soit, comme le mentionnent dans un euphémisme les auteurs de la notice, "pas toujours d'une précision académique ", c'est plus que l'exécution instrumentale, la qualité de l'improvisation vocale et instrumentale qui saisit l'auditeur, et non la rigueur des codes musicaux qui semblent n'avoir que peu d'importance. Par ailleurs, l'emploi du 'ûd pour la mise en valeur du texte, auquel s'ajoute parfois l'accompagnement rythmique du darbouka qui en souligne la cadence textuelle (plages 1, 7, 10,11, 15 et 17) marque bien la rupture avec la musique liturgique, essentiellement vocale, du culte synagogal. Suivent les piyyutim, constitués d'un nombre restreint de motifs mélodiques, qui prennent chez Yaacov Bchiri la forme de puissantes mélopées, tantôt nostalgiques, tantôt solennelles et tantôt joyeuses (plage 1, 2, 11), d'incantations ou de chants de louanges (plage 3, 7), de psalmodies et d'invocations (plage 10).

5 Les pièces instrumentales liturgiques et paraliturgiques présentées ici sont jouées en dehors du culte synagogal et du jour du shabbat (et, qui plus est, l'enregistrement a été effectué hors situation). Autant d'occasions qui permettent l'évocation des grands thèmes profanes et bibliques : à l'occasion des mariages (plage 6), le chantre peut ainsi évoquer selon l'humeur et les circonstances, des conseils aux mariés (plage 5), ou l'identification de l'attente de la bien-aimée à celle du messie (plage 1); dans une belle adaptation des actions de grâce (Birkat Hamazon) après le repas, il peut se livrer à une évocation litanique des figures tutélaires du judaïsme (plage 7); lorsqu'il entonne sa version d'une pièce maîtresse de la liturgie juive, le Kaddish (plage 8), il raconte la révolte des esclaves dans une saisissante hymne a cappella (plage 9); lors des processions du pèlerinage de la Ghriba (plage11), il narre le lien mythique entre Djerba et Jérusalem; il entonne également un chant le jeudi soir précédant la cérémonie de maturité religieuse des jeunes juifs djerbiens (plage 17).

6 Une seconde série de chants est liée au Shabbat, moment agrémenté de nombreux piyuttim prescrits ou spontanés. Ils doivent sans doute ici leur accompagnement 
instrumental à la performance hors contexte pour les besoins de l'enregistrement. Les thèmes abordés, qui varient "au gré de l'humeur du chanteur", sont la nostalgie de la Terre promise (plage 2), l'attente messianique (plage 3), l'identification de la fiancée au shabbat - chant solennel a cappella, généralement responsorial, mais repris ici par une seule voix (plage 4), la célébration de la mémoire d'un saint vénéré, psalmodiée en arabe et en hébreu (plage 10), ou encore la signification du jour du repos (plage 15).

Enfin, les pièces plus légères extraites du répertoire " populaire » du chantre (plage 13, $14,16,18)$, sont chantées en arabe pour les trois premières, et en arabe mêlé de français pour la dernière, bien que la structure musicale ne les distingue pas vraiment des autres. Il peut s'agir d'une thématique sociale, comme la liberté des femmes (plage 16) ou d'une romance des années 1920 (plage 18). Sans parler d'une adaptation tout à fait personnelle de l'hymne national israélien (plage 12), lui-même inspirée de la Moldau, qui est peut-être l'exemple le plus représentatif de réappropration d'éléments extérieurs dans son répertoire.

On peut formuler encore quelques remarques sur le livret, dont le lecteur appréciera à juste titre la finesse et la richesse d'informations sur la communauté djerbienne, qui témoignent d'un travail exigeant et passionné. On mentionnera tout de même deux erreurs : d'abord, la mélopée en arabe enseignée à Enrico Macias, signalée à la page 9, est indiquée "plage 19 ", mais il faut probablement lire «plage 15 »; toujours à la page 9 , l'auteur prétend qu'il n'existe de Yaacov Bchiri que ce CD et une « cassette bricolée », ils oublient de mentionner - rassurons l'amateur ! - un CD complet consacré a Bchiri (Yacoub B'chiri: Jewish and Arabic Songs from Jerba. Syncoop Produkties 5759 CD 235, enregistré deux ans avant celui-ci). Plus ennuyeux pour accéder à la matière musicale, on regrette l'absence de notes concernant d'une part le tambour darbouka (dont même le nom du musicien semble oublié !), d'autre part l'influence du contexte de l'enregistrement sur la performance instrumentale elle-même, notamment des textes liturgiques. En négligeant une description des pièces au niveau des modes mélodiques, des structures rythmiques et des piyyutim au profit des motifs narratifs, les auteurs prennent le risque de faire manquer à l'auditeur la pleine saveur des différentes pièces, dont l'ordre apparaît d'ailleurs aléatoire. La compréhension de certains textes aurait sans doute été enrichie par une transcription - voire une traduction - d'au moins quelques textes. L'épaisseur des livrets n'étant certes pas extensibles à l'infini, il est bien entendu que ces quelques réserves ne nuisent en rien à l'excellente qualité documentaire, artistique et éditoriale de ce CD.

\section{BIBLIOGRAPHIE}

ROTEN Hervé, 1998, Musiques liturgiques juives. Parcours et escales. Paris : Cité de la musique/ Arles : Actes Sud, collection « Musiques du Monde ».

SHILOAH Amnon, 1995, Les traditions musicales juives. Paris : Maisonneuve et Larose. 